\title{
$\mathrm{IV}$ 족 천이금속 질화물과 bcc Fe간 계면 에너지의 제일원리 연구
}

\author{
징순호 ${ }^{\dagger}$ - 정욱상 - 변지형 \\ 한간과과하기순연
}

\section{A First Principles Calculation of the Coherent Interface Energies between Group IV Transition Metal Nitrides and bcc Iron}

\author{
Soon-Hyo Chung ${ }^{\dagger}$, Woo-Sang Jung and Ji-Young Byun
}

Materials Science \& Technology Division, Korea Institute of Science \& Technology, Seoul 136-791, Korea

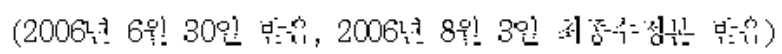

\begin{abstract}
The coherent interface energies and misfit strain energies of $\mathrm{Fe} / \mathrm{XN}$ (X=Ti, $\mathrm{Zr}, \mathrm{Hf}$ ) systems were calculated by first principles method. The interface energies in $\mathrm{Fe} / \mathrm{TiN}, \mathrm{Fe} / \mathrm{ZrN}$ and $\mathrm{Fe} / \mathrm{HfN}$ systems were 0.343 , 0.114 , and $0.030 \mathrm{~J} / \mathrm{m}^{2}$, respectively. Influence of bond energy was estimated using the discrete lattice plane/ nearest neighbor broken bond(DLP/NNBB) model. It was found that the dependence of interface energy on the type of nitride was closely related to changes of the bond energies between $\mathrm{Fe}, \mathrm{X}$ and $\mathrm{N}$ atoms before and after formation of the $\mathrm{Fe} / \mathrm{XN}$ interfaces. The misfit strain energies in $\mathrm{Fe} / \mathrm{TiN}, \mathrm{Fe} / \mathrm{ZrN}$, and $\mathrm{Fe} / \mathrm{HfN}$ systems were $0.239,1.229$, and $0.955 \mathrm{eV}$ per 16 atoms(Fe; 8 atoms and $\mathrm{XN} ; 8$ atoms). More misfit strain energy was generated as the difference of lattice parameters between the bulk $\mathrm{Fe}$ and the bulk XNs increased.
\end{abstract}

Key words Coherent interface energy, Misfit strain energy, Transition metal nitrides, boc iron, First principles calculation.

\section{1. 서 론}

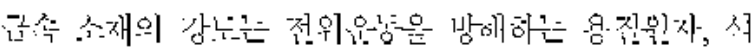



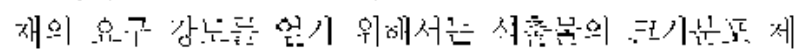

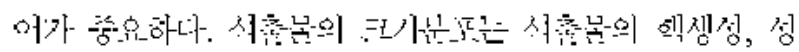

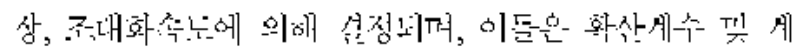

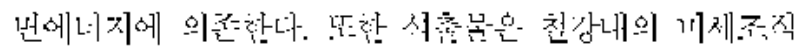

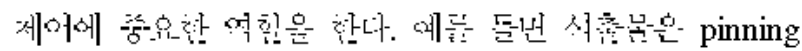
입자곤서 선정립 싱상을 어체하거나, 변테시의 웅선 헥


ㄴ:지가 충함하나.

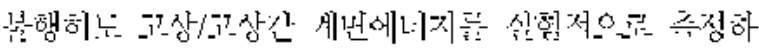

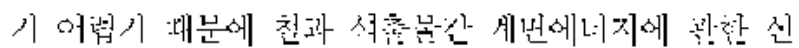

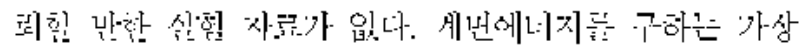

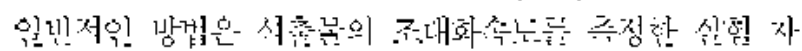



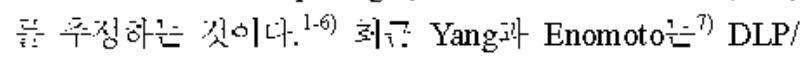
$\mathrm{NNBB}$ (discrete lattice plane/nearest neighbour broken

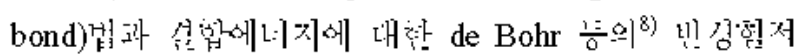

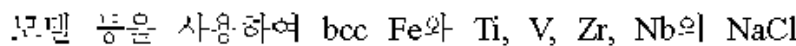

${ }^{\dagger}$ E-Mail : shompl@kist.re.kr

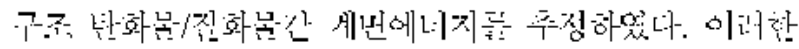

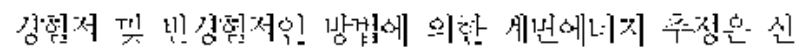



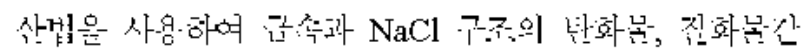

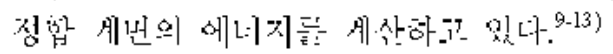

븐 연 구에서는 세인윈리례사나기빕을 이영 하여 bcc Fe와

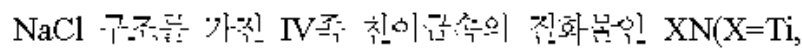

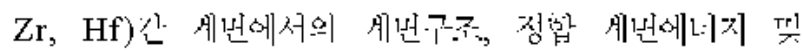

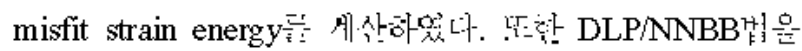

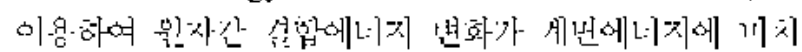
근 영힝:을 해서하했싸.

\section{2. 계산 방법}

\section{1 제일원리 계산}

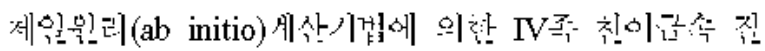

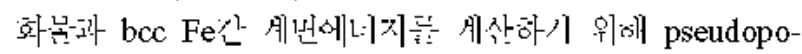

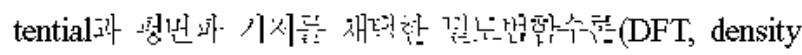
functional theory)베 계산은 행하었다. Perdew와 Wang에 ${ }^{14}$

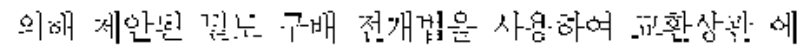

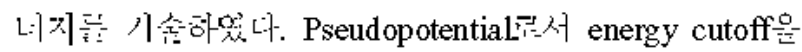

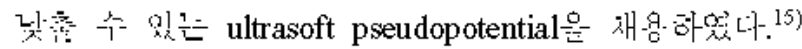

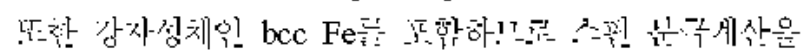


행하였다.

$\mathrm{XN}$ 상은 $\mathrm{NaCl}$ 형 구조이다. 또한 $\mathrm{Ti}-\mathrm{N}$ 계 질화물로서 tetragonal (rutile형) 구조의 $\mathrm{Ti}_{2} \mathrm{~N}$ 과 hexagonal 구조의 $\eta$ 상 $\left(1066^{\circ} \mathrm{C}\right.$ 이상에서 안정 $)$ 이 존재한다. $\mathrm{Hf}-\mathrm{N}$ 계 질화물로 서 hexagonal 구조의 $\mathrm{Hf}_{3} \mathrm{~N}_{2}, \mathrm{Hf}_{4} \mathrm{~N}_{3}$ 상 $\left(1200^{\circ} \mathrm{C}\right.$ 이상에서 안정)이 존재한다.

$\mathrm{XN}$ 상과 bcc $\mathrm{Fe}$ 간에는 식 (1)과 같은 Baker-Nutting 관계를 만족하는 반정합계면이 존재한다.

$$
\{001\}_{\mathrm{XN}} / /\{001\}_{\mathrm{Fe}} \text { 및 }<110>_{\mathrm{XN}} / /<100>_{\mathrm{Fe}}
$$

본 연구에서는 $\mathrm{Fe} / \mathrm{XN}$ 정합 계면 형성에 따른 정합계 면 에너지와 misfit strain energy를 계산하였다. 본 계산 에서는 작은 supercell의 사용을 허용하기 위해 이상적인 $1: 1$ 당량을 사용하였다. 이때 계면은 Fig. 1과 같은 bcc $\mathrm{Fe}$ 층과 $\mathrm{XN}$ 층으로 이루어진 supercell에 의해 모사된다. Supercell 내의 총원자수를 16 개 및 32 개로 하여 계의 총 에너지를 구한다. 이때 계의 에너지를 최소화하기 위한 구 조완화 계산을 행하고, supercell의 체적, 형상 및 supercell 내의 원자위치를 변화시킨다(상세한 계산 방법은 [16] 참 조).

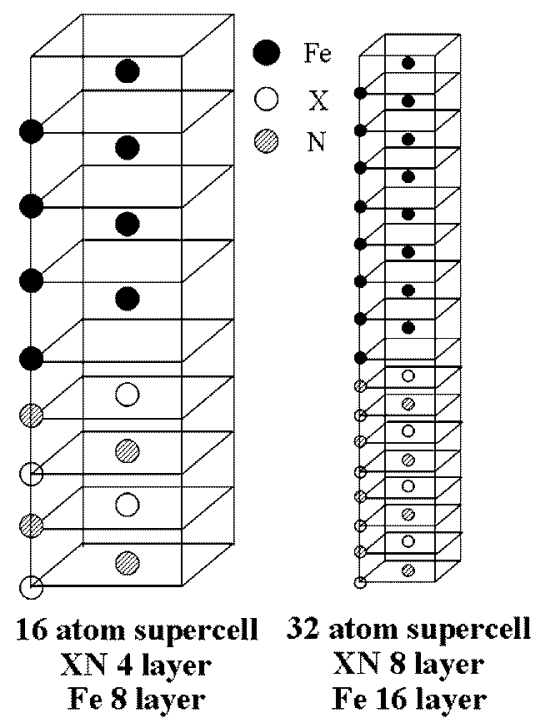

Fig. 1. Interface supercell structures for $\mathrm{Fe} / \mathrm{XN}$.

\subsection{DLP/NNBB법에 의한 계면에너지 계산}

본 연구에서는 제일원리로 계산한 값과 Yang과 Enomoto에 ${ }^{7)}$ 의해 제시된 DLP/NNBB법으로 계산된 계 면에너지 값을 비교해 보고자 하였다. DLP/NNBB법에 의하면 계면에너지, $\gamma_{N N B B}$ 는 근사적으로 다음 식으로 표 현할 수 있다.

$$
\begin{aligned}
& \gamma_{N N B B}=\left(e_{F e-N}^{\mathrm{NaCl} \mathrm{FeN}}-e_{X-N}^{\mathrm{NaCl} X N}\right) \\
& +\left[\left(4 e_{F e 1-X}^{b c c F e X}+e_{F e 2-X}^{b c c F e X}\right)\right. \\
& \left.-\left(2 e_{F e-F e, 1 N N}^{b c c F e}+0.5 e_{F e-F e, 2 N N}^{b c c F e}\right)-2 e_{X-X}^{f c c X}\right]
\end{aligned}
$$

여기서, $e_{\mathrm{Fe}-\mathrm{NaCl} F \mathrm{~N}}^{\mathrm{NaCl} X N}, e_{X-N}^{\mathrm{NaCl}}, e_{\mathrm{Fe}-X}^{b c c \mathrm{FeX}}, e_{\mathrm{Fe}-\mathrm{Fe}}^{b c c \mathrm{Fe}}, e_{X-X}^{f c c X}$ 는 각각 $\mathrm{NaCl}$ 구조의 $\mathrm{FeN}$ 에서의 $\mathrm{Fe}-\mathrm{N}$ 결합, $\mathrm{NaCl}$ 구조 $\mathrm{XN}$ 에서의 X-N 결합, $\mathrm{bcc} \mathrm{FeX}$ 에서의 $\mathrm{Fe}-\mathrm{X}$ 결합, $\mathrm{bcc}$ $\mathrm{Fe}$ 에서의 $\mathrm{Fe}-\mathrm{Fe}$ 결합, $\mathrm{XN}$ 내 $\mathrm{fcc}$ 구조 $\mathrm{X}$ sublattice에서 의 X-X 결합의 에너지를 나타낸다. $e_{F e 1-X}^{b c c}, e_{F e 2-X}^{b c c F}$, 에서의 하첨자 $\mathrm{Fe}_{1}, \mathrm{Fe}_{2}$ 는 계면에서 첫 번째 및 두 번 째 원자층의 $\mathrm{Fe}$ 원자를 나타낸다. $e_{F e-F e}^{b c c}$ 에서의 하첨 자 $1 \mathrm{NN}$ 및 $2 \mathrm{NN}$ 은 bulk $\mathrm{Fe}$ 에서의 최인접원자간 및 두 번째 인접 원자간 거리를 나타낸다. 식 (2)에서 첫 번째 항은 X-N 반결합(half bond) 2개가 꼲어지고 Fe-N 결합 1 개가 형성될 때의 에너지 변화를 나타내고, 두 번째 항 은 $\mathrm{bcc} \mathrm{Fe}$ 에서의 $\mathrm{Fe}-\mathrm{Fe}$ 반결합 5개(최인접 4개 및 두 번째 인접 1개) 및 XN에서 X-X 반결합 4개가 긇어지 고 Fe-X 결합 5개(최인접 4개 및 두 번째 인접 1개)가 형성될 때의 에너지 변화를 나타낸다. 본 연구에서는 제 일원리계산에 의해 식 (2)의 결합에너지를 구하였다 (상 세한 계산 방법은 [16] 참조).

\section{3. 결과 및 고찰}

\subsection{Bulk 상 및 $\mathrm{Fe} / \mathrm{XN}$ 계의 격자상수}

Table 1에 제일원리를 이용하여 얻어진 bcc $\mathrm{Fe}, \mathrm{X}$ 및 $\mathrm{XN}$ 상의 격자상수와 bulk modulus를 문헌에 보고된 실 혐결과와 함께 보인다. ${ }^{17,18)}$ 격자상수의 계산치와 실험치 간의 차이는 $0.101 \AA$ 이하로 잘 일치하였다. 반면, bulk

Table 1. Lattice constants(a) and bulk modulus(B) of Fe, X and XN

\begin{tabular}{ccccccc}
\hline \hline & $a_{0 \mathrm{Cal}}(\AA)$ & $a_{0 \operatorname{Exp}}(\AA)$ & $a_{0 \mathrm{Cal}}-a_{0 \operatorname{Exp}}(\AA)$ & $B_{0 \mathrm{Cal}}(\mathrm{GPa})$ & $B_{0 \operatorname{Exp}}(\mathrm{GPa})$ & $B_{0 \mathrm{Cal}}-B_{0 \text { Exp }}(\AA)$ \\
\hline $\mathrm{Fe}$ & 2.860 & $2.867^{17}$ & -0.007 & 151 & $169.8^{17)}$ & -18.8 \\
$\mathrm{Ti}$ & 3.244 & $3.311^{17)}$ & -0.067 & 103 & $108.4^{17)}$ & -5.4 \\
$\mathrm{Zr}$ & 3.541 & $3.568^{17)}$ & -0.027 & 90 & $89.8^{17)}$ & 0.2 \\
$\mathrm{Hf}$ & 3.514 & $3.615^{17)}$ & -0.101 & 102 & $109^{17)}$ & -7 \\
$\mathrm{TiN}$ & 4.256 & $4.239^{17)}$ & 0.017 & 282 & $288^{18)}$ & -6 \\
$\mathrm{ZrN}$ & 4.594 & $4.585^{17)}$ & 0.009 & 265 & - & - \\
$\mathrm{HfN}$ & 4.522 & $4.530^{18)}$ & -0.008 & 275 & - & - \\
\hline
\end{tabular}


Table 2. Lattice constants of $F e / X N$ interface $\left(a_{X N}^{\prime}=a_{X N} / \sqrt{2}\right)$

\begin{tabular}{|c|c|c|c|c|c|c|c|c|}
\hline & $a_{X N}^{\prime}(\dot{\Lambda})$ & $\frac{a_{X N}^{\prime}}{a_{F e}}$ & $a_{F \mathscr{K} X N}(\dot{\Lambda})$ & $\frac{a_{F e / X N}-a_{F e}}{a_{X N}^{\prime}-a_{F e}}$ & $\frac{a_{F e / X N}}{a_{F e}}$ & $\frac{a_{X N}^{\prime}}{\sqrt{2}}(\Lambda)$ & $\frac{a_{F e} / X N}{\sqrt{2}}(\Lambda)$ & $\frac{a_{F e / X N}-a_{X N}^{\prime}}{\sqrt{2}}(\dot{\Lambda})$ \\
\hline $\operatorname{TiN}$ & 3.010 & 1.052 & 2.974 & 0.76 & 1.040 & 2.128 & 2.103 & -0.025 \\
\hline $\mathrm{ZrN}$ & 3.248 & 1.136 & 3.171 & 0.80 & 1.109 & 2.297 & 2.242 & -0.055 \\
\hline $\mathrm{HfN}$ & 3.198 & 1.118 & 3.124 & 0.78 & 1.092 & 2.261 & 2.209 & -0.052 \\
\hline
\end{tabular}



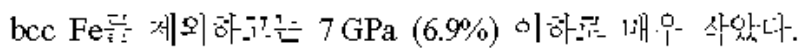

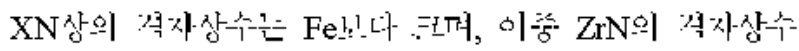

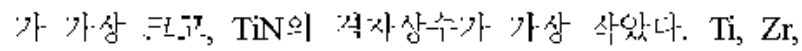

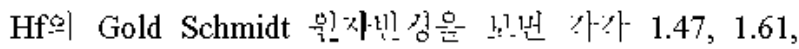

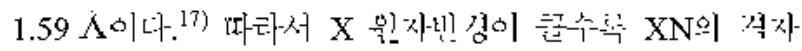

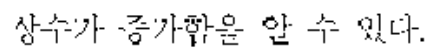

Table 2는 $\mathrm{Fe} / \mathrm{XN}$ 예의 격자상궁 셰산 션과이다. $\mathrm{XN}$ 의

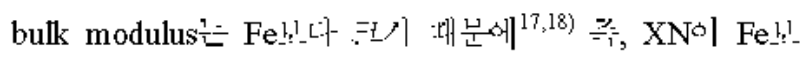
다 hard하이 대분에 $\mathrm{Fe} / \mathrm{XN}$ 예의 격자장수가 bulk Fe리나다 bulk XN에 다 가까운 값은 가젔다. 츤, $\left(a_{F e / X N}-a_{F e}\right)$ $/\left(a_{X N}-a_{F e}\right)$ 의 삾이 $0.76 \sim 0.80$ 이나. 이는 misfit 에 L:

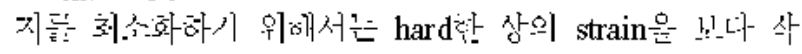
레 하여야 하기 대분이다.

\section{$3.2 \mathrm{Fe} / \mathrm{XN}$ 계의 계면 구조}

\subsection{1 $\mathrm{XN}$ 내 rippling}

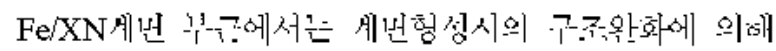

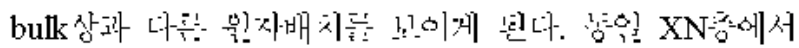

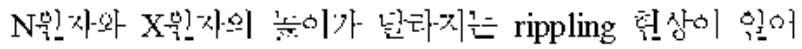
난다. Table 3 에 $\mathrm{Fe} / \mathrm{XN}$ 예의 $\mathrm{XN}$ 네 rippling 셰산견과ㄴㅡㅜㄴ

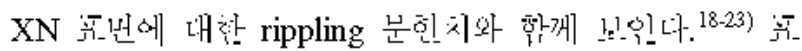

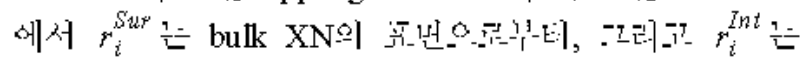



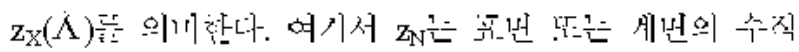

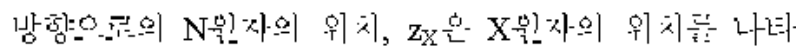

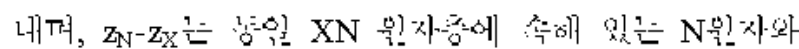

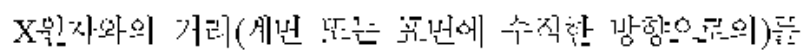

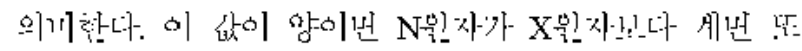
는 푸넌네에 가깝게 위치한은 의끼한다.

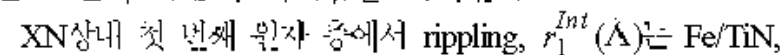
$\mathrm{Fe} / \mathrm{ZrN}, \mathrm{Fe} / \mathrm{HfN}$ 예에 내해 아아 $-0.103,-0.156,-0.224 \AA$

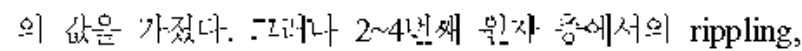

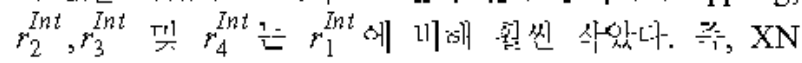
상에서의 rippling현 계넌 직하 $\mathrm{XN}$ 응에서 신하게 인어


의 rippling 정나는 응의 삾은 삿거 있으여, $\mathrm{TiN}, \mathrm{ZrN}$, $\mathrm{HfN}$ 은폰 천대치가 긍가한다.

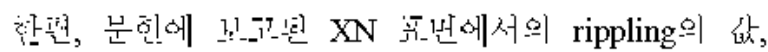



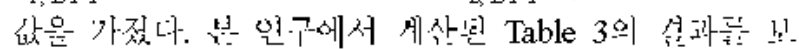

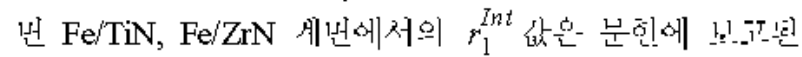

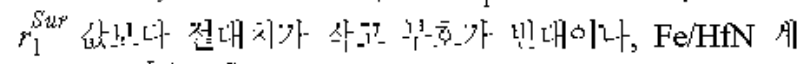
에서는 $r_{1}^{\text {Int }}, r_{1}^{S u r}$ 의 천내치가 뮤사하나. 픈, $\mathrm{Fe} / \mathrm{XN}$ 예

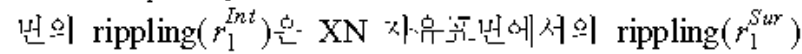

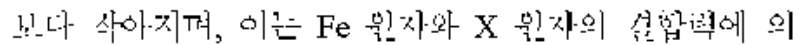

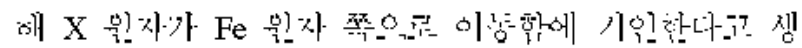




찬 니가 긍가하기 :대분에(Table 6의 (C)/(B) 찬죽) X가

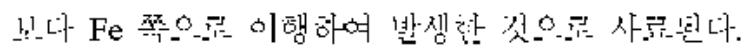

3.2.2 Fe/XN 계넌에서의 힉자인 선갑긴이(bond length)

Table 3. Rippling for $\mathrm{XN}$ surface and Fe-XN interface

\begin{tabular}{|c|c|c|c|c|c|c|}
\hline & \multicolumn{2}{|c|}{ XNN surface } & \multicolumn{4}{|c|}{$\mathrm{Fe} / \mathrm{XN}$ interface } \\
\hline & \multicolumn{2}{|c|}{ DFT (Previous Work) } & \multicolumn{4}{|c|}{ DFT (Present Work) } \\
\hline & $\begin{array}{c}S u \psi \\
r_{1, D F T}\end{array}$ & $S_{2, D F T}^{S u r}$ & $\begin{array}{l}I n t \\
r_{1, D F T}\end{array}$ & $r_{2, D F T}^{\text {Int }}$ & $\begin{array}{l}I n t \\
r_{3, D F T}\end{array}$ & $\begin{array}{l}I n t \\
r_{4, D F T}\end{array}$ \\
\hline $\operatorname{TiN}$ & $\begin{array}{l}0.156^{19)} \\
0.18^{20)} \\
0.178^{21)} \\
0.188^{22)} \\
0.179^{23)} \\
0.21^{18)}\end{array}$ & $\begin{array}{l}0.031^{21)} \\
0.026^{22)} \\
0.028^{23)} \\
0.005^{-18}\end{array}$ & -0.103 & 0.029 & 0.021 & -0.030 \\
\hline $\mathrm{ZrN}$ & $0.20^{18)}$ & $0.017^{18)}$ & -0.156 & 0.047 & 0.039 & -0.049 \\
\hline $\mathrm{HfN}$ & $0.21^{18)}$ & $0.022^{18)}$ & -0.224 & 0.049 & 0.033 & -0.065 \\
\hline
\end{tabular}


Table 4. Fe-N and Fe-X bond lengths $(\AA)$ at $\mathrm{Fe} / \mathrm{XN}$ interfaces

\begin{tabular}{cccccccccc}
\hline \hline 32 atoms & $\mathrm{Fe}_{1}-\mathrm{X}_{1}$ & $\mathrm{Fe}_{1}-\mathrm{N}_{1}$ & $\mathrm{X}_{1}-\mathrm{N}_{2}$ & $\mathrm{~N}_{1}-\mathrm{X}_{2}$ & $\mathrm{~N}_{2}-\mathrm{X}_{3}$ & $\mathrm{X}_{2}-\mathrm{N}_{3}$ & $\mathrm{X}_{1}-\mathrm{N}_{1}$ & $\mathrm{Fe}_{1}-\mathrm{Fe}_{2}$ & $\mathrm{X}_{1}-\mathrm{Fe}_{2}$ \\
\hline \multirow{2}{*}{$\mathrm{TiN}$} & 2.775 & 1.919 & 2.155 & 2.081 & 2.166 & 2.116 & 2.101 & 2.469 \\
& $(-0.07)^{* 1}$ & $(-0.144)^{*_{2}}$ & $(+0.027)^{* 3}$ & $(-0.047)^{* 3}$ & $(+0.038)^{* 3}$ & $(-0.012)^{* 3}$ & $(-0.027)^{* 3}$ & $(-0.008)^{*_{4}}$ & 3.110 \\
\multirow{2}{*}{$\mathrm{ZrN}$} & 2.891 & 1.985 & 2.355 & 2.245 & 2.366 & 2.280 & 2.245 & 2.544 & \\
& $(-0.106)^{* 1}$ & $(-0.078)^{* 2}$ & $(+0.058)^{* 3}$ & $(-0.052)^{* 3}$ & $(+0.069)^{* 3}$ & $(-0.017)^{* 3}$ & $(-0.052)^{* 3}$ & $(+0.067)^{* 4}$ & 3.031 \\
\multirow{2}{*}{$\mathrm{HfN}$} & 2.842 & 2.016 & 2.356 & 2.180 & 2.337 & 2.255 & 2.218 & 2.525 & \\
& $(-0.155)^{* 1}$ & $(-0.047)^{* 2}$ & $(+0.095)^{* 3}$ & $(-0.08)^{* 3}$ & $(+0.076)^{* 3}$ & $(-0.006)^{* 3}$ & $(-0.043)^{* 3}$ & $(+0.048)^{* 4}$ & 3.015 \\
\hline
\end{tabular}

*1 Difference between $\mathrm{Fe}_{1}-\mathrm{X}_{1}$ bond lengths at relaxed and unrelaxed interfaces

*2 Difference between the $\mathrm{Fe}_{1}-\mathrm{N}_{1}$ bond lengths and the $\mathrm{Fe}-\mathrm{N}$ bond length $(2.063 \AA)$ at bulk FeN

*3 Difference between X-N bond lengths at the relaxed interfaces and the bulk XNs.

*4 Difference between the $\mathrm{Fe}_{1}-\mathrm{Fe}_{2}$ bond lengths and the $\mathrm{Fe}-\mathrm{Fe}$ bond length $(2.477 \AA)$ at bulk bcc $\mathrm{Fe}$

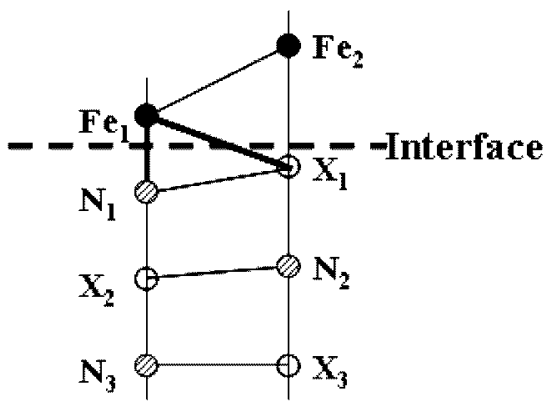

Fig. 2. Atomic structures at the $\mathrm{Fe} / \mathrm{XN}$ interface.

Table 4에 $\mathrm{Fe} / \mathrm{XN}$ 계면에서의 원자간 결합길이를 보인 다. 여기서 하첨자 1,2 는 계면에서 1,2 번째 원자층을 의미한다. Fig. 2는 계면근방 원자배치의 도식도이다.

$\mathrm{XN}$ 내 1 번째 원자 층 내의 $\mathrm{X}$ 와 N원자간 거리, $\mathrm{d}_{\mathrm{X} 1 \mathrm{~N} \text { 은 }}$ $\left[\left(a_{\mathrm{Fe} / X N} / \sqrt{2}\right)^{2}+\left(r_{1}^{I n t}\right)^{2}\right]^{0.5}$ 로 주어친다. Rippling 정도 $r_{1}^{I n t}$ 값은 $0.224 \AA$ 이하로 작기 때문에 $\mathrm{d}_{\mathrm{X} 1 \mathrm{~N} 1}$ 은 $a_{\mathrm{Fe} / \mathrm{XN}} / \sqrt{2}$ 와 거의 일치한다. 따라서, 계면 형성시의 $\mathrm{d}_{\mathrm{X1}-\mathrm{N} 1}$ 크기 변화, $\left|\mathrm{d}_{\mathrm{X} 1-\mathrm{N} 1}-a_{\mathrm{XN}}^{\prime} / \sqrt{2}\right|$ 은 $\mathrm{XN}$ 상의 격자상수 변화 $\left|a_{\mathrm{Fe} X N}-a_{\mathrm{XN}}^{\prime}\right| /$ $\sqrt{2}$ 와 유사하다(Table 3). 계면에서의 $\mathrm{Fe}_{1}-\mathrm{N}_{1}$ 결합길이 $\left(\mathrm{d}_{\mathrm{Fel}-\mathrm{N} 1}\right)$ 는 bulk $\mathrm{FeN}$ 에서의 결합길이보다 작다. $\mathrm{Fe}_{1}-\mathrm{X}_{1}$ 결합길이 $\left(\mathrm{d}_{\mathrm{Fel}-\mathrm{X} 1}\right)$ 는 $\left[\left(a_{\mathrm{FeXN}} / \sqrt{2}\right)^{2}+\left(\mathrm{d}_{\mathrm{Fel}-\mathrm{Nl}}+r_{1}^{I n t}\right)^{2}\right]^{0.5}$ 로 표 시된다. $r_{1}^{I n t}$ 가 음의 값을 갖고 있기 때문에, $\mathrm{d}_{\mathrm{Fe} 1 \mathrm{X} 1}$ 값은 rippling이 없는 경우보다 작아진다. 그리고 계면에서의 $\mathrm{Fe}_{1}-\mathrm{X}_{1}$ 결합길이 $\left(\mathrm{d}_{\mathrm{Fel}-\mathrm{Xl}}\right)$ 값은 제일원리에 의해 계산된 가 상적인 bcc Fe-X 격자에서의 최인접 원자간거리 $\left(\sqrt{3} a_{\mathrm{FeX}} /\right.$ 2, $2.554 \AA$ (bcc Fe-Ti), $2.733 \AA$ (bcc Fe-Zr), $2.702 \AA$ (bcc Fe-Hf)) 보다 더 길어진다. 이는 $\mathrm{d}_{\mathrm{Fel}-\mathrm{N} 1}+r_{1}^{I n t}$ 값이 $a_{\mathrm{FeX}} / 2$ 보다 크다는 사실에 기인한다. $\mathrm{Fe} / \mathrm{TiN}$ 계에서 $\mathrm{Fe}_{1}-\mathrm{Fe}_{2}$ 결합길이는 bulk Fe에서의 결합길이 $\left(\sqrt{3} a_{\mathrm{F}} 2\right)$ 보다 짧다. 반면 $\mathrm{Fe} / \mathrm{ZrN}, \mathrm{Fe} / \mathrm{HfN}$ 계에서는 bulk $\mathrm{Fe}$ 에서보다 약간 길 다.

첫 번째와 두 번째 $\mathrm{Fe}$ 층간 간격 $\mathrm{z}_{\mathrm{Fe} 2}-\mathrm{Z}_{\mathrm{Fe} 1}$ 는 $\mathrm{Fe} / \mathrm{TiN}$, $\mathrm{Fe} / \mathrm{ZrN}, \mathrm{Fe} / \mathrm{HfN}$ 계에서 각각 $1.294,1.202,1.223 \AA$ 이 며 이는 $\mathrm{bcc} F \mathrm{Fe}$ 격자에서의 층간간격 $\left(a_{\mathrm{Fe}} / 2=1.43 \AA\right)$ 보다 작다. $\mathrm{X}_{1}-\mathrm{Fe}_{2}$ 결합길이 $\left(\mathrm{d}_{\mathrm{X} 1-\mathrm{Fe} 2}\right)$ 는 $\left(\mathrm{Z}_{\mathrm{Fe} 1}-\mathrm{Z}_{\mathrm{X} 1}\right)+\left(\mathrm{Z}_{\mathrm{Fe} 2}-\mathrm{Z}_{\mathrm{Fe} 1}\right)=$ $\left(\mathrm{d}_{\mathrm{Fel}-\mathrm{N} 1}+r_{1}^{I n t}\right)+\mathrm{Z}_{\mathrm{Fe} 2}-\mathrm{Z}_{\mathrm{Fe} 1}$ 로 주어진다. $\mathrm{Z}_{\mathrm{Fel}}-\mathrm{Z}_{\mathrm{X} 1}$ 값은 $\mathrm{Fe} / \mathrm{TiN}$, $\mathrm{Fe} / \mathrm{ZrN}, \mathrm{Fe} / \mathrm{HfN}$ 계에서 각각 $1.816,1.829,1.792 \AA$ 을 갖 고 이 값은 가상적인 bcc Fe-X 격자에서의 $a_{\mathrm{FeX}} / 2(\mathrm{Fe} /$ $\mathrm{TiN}, \mathrm{Fe} / \mathrm{ZrN}, \mathrm{Fe} / \mathrm{HfN}$ 계에서 각각 $1.474,1.578$, $1.560 \AA$ )보다 크다. $\mathrm{Fe} / \mathrm{XN}$ 계는 bulk $\mathrm{XN}$ 에 비해 $\mathrm{X}_{2}-\mathrm{N}_{3}$, $\mathrm{N}_{1}-\mathrm{X}_{2}$ 결합길이는 짧고 $\mathrm{N}_{2}-\mathrm{X}_{3}, \mathrm{X}_{1}-\mathrm{N}_{2}$ 결합길이는 더 길 다.

\section{3 $\mathrm{Fe} / \mathrm{XN}$ 몌의 계면에너지 및 misfit strain energy}

\subsection{1 $\mathrm{Fe} / \mathrm{XN}$ 계의 계면에너지}

$\mathrm{Fe} / \mathrm{XN}$ 계의 계면에너지 계산 결과를 Table 5 에 나타내 었다. $\mathrm{Fe} / \mathrm{XN}$ 계면에너지는 $0.03 \sim 0.343 \mathrm{~J} / \mathrm{m}^{2}$ 의 값을 보인 다. Dudiy 등 ${ }^{10,13)}$ 은 제일원리계산을 통해 $\mathrm{Co} / \mathrm{TiN}, \mathrm{Cu} / \mathrm{TiN}$, $\mathrm{Al} / \mathrm{TiN}, \mathrm{Ti} / \mathrm{TiN}$ 의 계면에너지가 각각 $0.22,1.01,0.62$ $0.94,-0.48 \mathrm{~J} / \mathrm{m}^{2}$ 임을 보고하였다. $\mathrm{Fe}$ 보다 원자번호가 하 나 큰 $\mathrm{Co} / \mathrm{TiN}$ 계의 계면에너지 $\left(0.22 \mathrm{~J} / \mathrm{m}^{2}\right)$ 는 본 연구의 $\mathrm{Fe} / \mathrm{TiN}$ 에서의 $0.343 \mathrm{~J} / \mathrm{m}^{2}$ 보다 약간 낮다.

계면 형성시 원자간 결합에너지 변화가 계면에너지에

Table 5. Interfacial and misfit strain energies of $\mathrm{Fe} / \mathrm{XN}$ systems

\begin{tabular}{cccccc}
\hline \hline \multirow{2}{*}{ System } & $\gamma_{\text {ib initio }}$ & \multicolumn{4}{c}{ Misfit strain energy } \\
\cline { 3 - 6 } & $\left(\mathrm{J} / \mathrm{m}^{2}\right)$ & $\begin{array}{c}\text { Total, A } \\
(\mathrm{eV} / 16 \text { atoms })\end{array}$ & $\begin{array}{c}\text { Fe Phase, B } \\
(\mathrm{eV} / 8 \text { atoms })\end{array}$ & $\begin{array}{c}\text { XN Phase, C } \\
(\mathrm{eV} / 8 \text { atoms })\end{array}$ & $\begin{array}{c}\text { Error, A-(B+C) } \\
(\mathrm{eV} / 16 \mathrm{Catoms})\end{array}$ \\
\hline $\mathrm{Fe} / \mathrm{TiN}$ & 0.343 & 0.239 & 0.135 & 0.077 & 0.027 \\
$\mathrm{Fe} / \mathrm{ZrN}$ & 0.114 & 1.229 & 0.959 & 0.273 & -0.003 \\
$\mathrm{Fe} / \mathrm{HfN}$ & 0.030 & 0.955 & 0.717 & 0.281 & -0.043 \\
\hline
\end{tabular}


Table 6. Interfacial energies of Fe/XN systems estimated by NNBB model

\begin{tabular}{|c|c|c|c|c|c|c|c|c|c|}
\hline & $\begin{array}{c}(\mathrm{A}), \mathrm{eV} \\
-e_{\mathrm{Fe}-\mathrm{N}}^{\mathrm{NaCl} F \mathrm{~F}}\end{array}$ & $\begin{array}{c}\text { (B), eV } \\
-e_{X-N}^{N \alpha C i X N}\end{array}$ & $\begin{array}{c}(\mathrm{C}), \mathrm{eV} \\
-\left(4 e_{F e 1-X}^{b c c F e X}\right. \\
\left.+-e_{F e 2 X}^{b c c F e X}\right)\end{array}$ & $\begin{array}{l}\text { (D), eV } \\
-2 e_{X-X}^{f c c} X\end{array}$ & $\begin{array}{c}(\mathrm{E}), \mathrm{eV} \\
-\left(2 e_{F e-F e, 1 N N}^{b c c-F e}\right. \\
\left.+0.5 e_{F e-F e, 2 N N}^{b c c F e}\right)\end{array}$ & $\begin{array}{c}-(\mathrm{A})+(\mathrm{B}) \\
\mathrm{eV}\end{array}$ & $\begin{array}{l}-(\mathrm{C})+(\mathrm{D}) \\
+(\mathrm{E}), \mathrm{eV}\end{array}$ & $\begin{array}{c}\gamma_{N N B B} \\
(\mathrm{eV} / \\
\text { 2atomXN) }\end{array}$ & $\begin{array}{l}\gamma_{N N B B} \\
\left(\mathrm{~J} / \mathrm{m}^{2}\right)\end{array}$ \\
\hline $\mathrm{Fe} / \mathrm{TiN}$ & 0.890 & 1.458 & 3.841 & 1.650 & 1.802 & 0.568 & -0.390 & 0.178 & 0.324 \\
\hline $\mathrm{Fe} / \mathrm{ZrN}$ & 0.890 & 1.459 & 4.306 & 2.046 & 1.802 & 0.569 & -0.459 & 0.110 & 0.175 \\
\hline $\mathrm{Fe} / \mathrm{HfN}$ & 0.890 & 1.477 & 4.405 & 2.089 & 1.802 & 0.587 & -0.514 & 0.073 & 0.120 \\
\hline
\end{tabular}

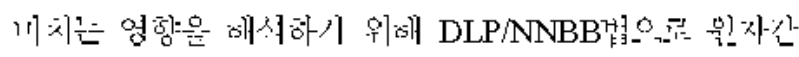

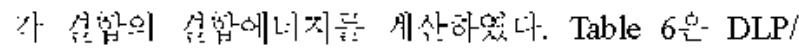

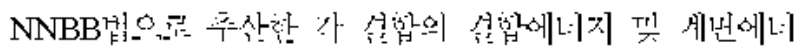
지 셰산 셜과이다. $\mathrm{Fe} / \mathrm{TiN}$ 예에서는 훅산한 셰넌에L:지

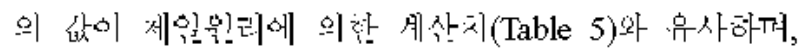

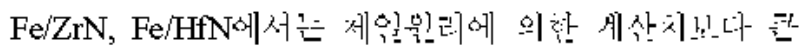

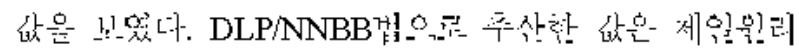

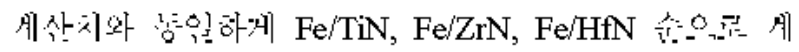
넌에니치가 산소하었다.

$\mathrm{Fe} / \mathrm{TiN}, \mathrm{Fe} / \mathrm{ZrN}, \mathrm{Fe} / \mathrm{HfN}$ 에서 $\mathrm{Fe}-\mathrm{N}$ 건햅에니지의 전내 치근 $0.890 \mathrm{eV}$ 이며, $\mathrm{X}-\mathrm{N}$ 권합에 $\mathrm{L}: \mathrm{l}$ 지의 천대치는 차아

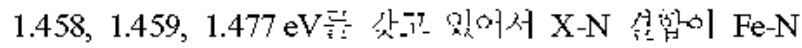

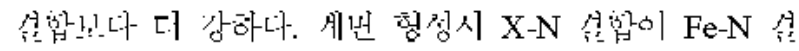

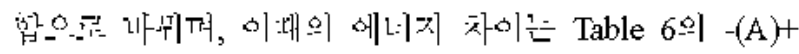
(B)항에 닌인 나와 간시 태분에 $\mathrm{Fe} / \mathrm{TiN}$ 낒 $\mathrm{Fe} / \mathrm{ZrN}$ 계의 셰넌엔:지아 $\mathrm{Fe} / \mathrm{HAN}$ 세닌다 닊은 것이 에상린다. 한편 $\mathrm{X}-\mathrm{X}$ 낒 $\mathrm{Fe}-\mathrm{X}$ 선합에 $\mathrm{t}: \mathrm{x}$ 지의 전대치는 $\mathrm{Fe} / \mathrm{TiN}, \mathrm{Fe} / \mathrm{ZrN}$,

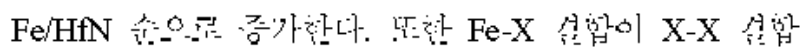
닌다 더 강하기 대분에 식 (2)의 두놘째 항에 해낭하느는

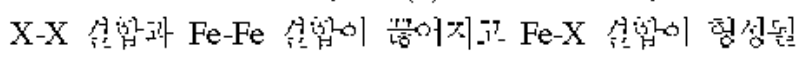

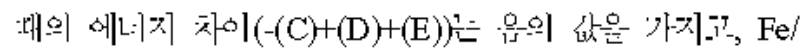

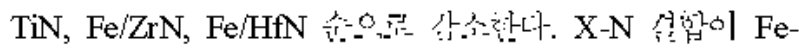

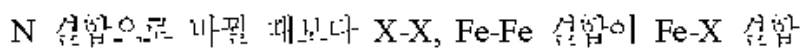

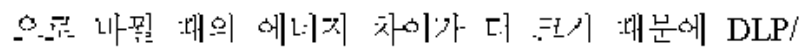

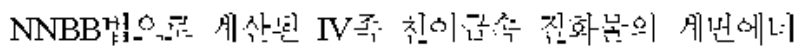
지는 $\mathrm{Fe} / \mathrm{HfN}$ 예가 가상 삭겅, $\mathrm{Fe} / \mathrm{ZrN}$ 예, $\mathrm{Fe} / \mathrm{TiN}$ 예 돈 긍아-한다.

\subsection{2 $\mathrm{Fe} / \mathrm{XN}$ 셰의 misfit strain energy}



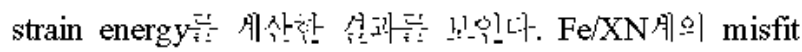
strain energy아 $\mathrm{Fe}$ 상과 $\mathrm{XN}$ 상의 misfit strain energy 합과 의 차는 $\mathrm{Fe} / \mathrm{TN}, \mathrm{Fe} / \mathrm{ZrN}, \mathrm{Fe} / \mathrm{HfN}$ 에서 하아 $0.027,-0.003$, $-0.043 \mathrm{eV} / 16$ atom.롤 1 배웅 삭다. 따라서 $\mathrm{Fe} / \mathrm{XN}$ 예의 misfit strain energy는 아상의 시여눈은 셰산ㅎㅎㅇㅕ 그 든은 합하

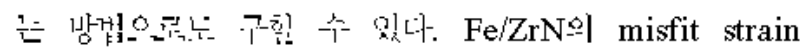

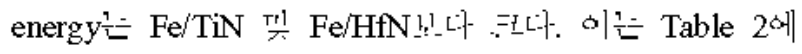
는이는 나아 같이 misfit $\left(a_{F e, \alpha N} / a_{F e}\right)$ 이 $\mathrm{Fe} / \mathrm{TiN}, \mathrm{Fe} / \mathrm{ZrN}, \mathrm{Fe} /$ $\mathrm{HfN}$ 에서 나아 $1.040,1.109,1.092$ 폰서 $\mathrm{Fe} / \mathrm{ZrN}$ 예가 가
상 조기 :대분이다.

\section{4. 결 론}

IV폭 칙이걸숙 진화논 TiN, ZrN, HAN 이 bcc Fe와

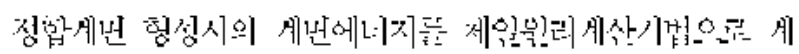

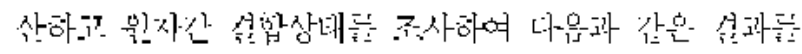
얻엤다.

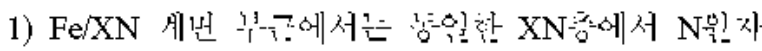

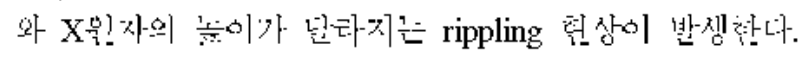


은 가지며 TiN, $\mathrm{ZrN}, \mathrm{HfN}$ 슨을 전대치가 긍가한다.

2) 제인은리네 계산은 항-해 얻현 $\mathrm{Fe} / \mathrm{TiN}, \mathrm{Fe} / \mathrm{ZrN}, \mathrm{Fe} / \mathrm{HfN}$ 셰넌의 셰넌에니지는 아아 $0.343,0.114,0.030 \mathrm{~J} / \mathrm{m}^{2}$ 이있 ㄴ:-).

3) $\mathrm{Fe} / \mathrm{XN}$ 셰넌엔:지에 영힝:운 끼치는 화학션



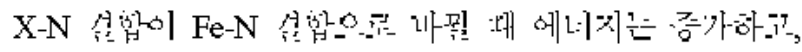

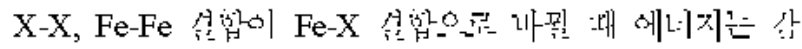
소하였다.

4) $\mathrm{Fe} / \mathrm{TiN}$ 예, $\mathrm{Fe} / \mathrm{ZrN}$ 예, $\mathrm{Fe} / \mathrm{HfN}$ 예의 misfit strain energy 는 나아 $0.239,1.229,0.955 \mathrm{eV} / 16$ atoms 이있다.

\section{감사의 글}

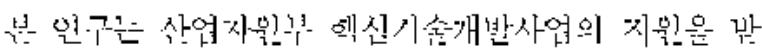

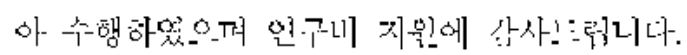

\section{참 고 문 헌}

1. D. A. Porter and K. E. Easterling, Phase Transformation in Metals and Alloys, 2nd Ed. Chapman \& Hall, London, UK, (1992).

2. J. D. Robson and H. K. D. H. Bhadeshia, Calphad, 20, 447 (1996).

3. N. Fujita and H. K. D. H. Bhadeshia, Mater. Sci. Technol., 17, 403 (2001).

4. N. Fujita and H. K. D. H. Bhadeshia, ISIJ Inter., 42, 760 (2002).

5. N. Fujita, H. K. D. H. Bhadeshia and M. Kikuchi, 
Modelling Simul. Mater. Sci. Eng., 12, 273 (2004).

6. J. Hald and L. Korcakova, ISIJ Inter., 43, 420 (2003).

7. Z. -G. Yang and M. Enomoto, Mater. Sci. Eng., A332, 184 (2002).

8. F. R. de Boer, R. Boom, W. C. M, Mattens, A. R. Miedema and A. K. Niessen, Cohesion in Metals; Transition Metal Alloys, North-Holland, Amsterdam, (1988).

9. J. Hartford, Phys. Rev. B, 61, 2221 (2000).

10. S. V. Dudiy and B. I. Lundqvist, Phys. Rev. B, 64, 045403 (2001).

11. M. Christensen, S. Dudiy and G. Wahnstroem, Phys. Rev. B, 65, 045408 (2002).

12. D. J. Siegel, L. G. Hector and J. B. Adams, Phys. Rev. B, 67, 092105 (2003).

13. S. V. Dudiy and B. I. Lundqvist, Phys. Rev. B, 69, 125421 (2004).

14. J. P. Perdew, Electronic Structure of Solids, eds. P. Ziesche and H. Eschrig, Akademie Verlag, Berlin, (1991).

15. K. Laasonen, A. Pasquarello, R. Car, C. Lee and D. Vanderbilt, Phys. Rev. B, 47, 10142 (1993).

16. W. S. Jung, S. H. Chung and J. Y. Byun, J. Kor. Inst. Met. \& Mater., 43, 626 (2005).

17. Smithells Metals Reference Book, eds. W. F. Gale and T. C. Totemeier, Elsevier, Amsterdam, (2004).

18. K. Kobayashi, Surf. Sci., 493, 665 (2001).

19. L. M. Liu, S. Q. Wang and H. Q. Ye, J.Phys. Condens. Matter, 15, 8103 (2003).

20. D. Gall, S. Kodambaka, M. A. Wall, I. Petrov and J. E. Greene, J. Appl. Phys., 93, 9086 (2003).

21. N. C. Hemandez, J. Graciani and J. F. Sanz, Surf. Sci., 541, 217 (2003).

22. N. C. Hemandez and J. F.Sanz, Int. J. Mol. Sci., 2, 263 (2001).

23. M. Marlo and V. Milman, Phys. Rev. B, 62, 2899 (2000). 